\title{
Two-stage Kondo effect in side-coupled quantum dots: Renormalized perturbative scaling theory and Numerical Renormalization Group analysis
}

\author{
Chung-Hou Chung ${ }^{1},{ }^{3}$, Gergely Zarand ${ }^{1},{ }^{2}$, and Peter Wölfle ${ }^{1},{ }^{4}$ \\ 1 Institut für Theorie der Kondensierten Materie, \\ Universität Karlsruhe, 76128 Karlsruhe, Germany \\ 2 Department of Theoretical Physics, Institute of Physics, \\ Budapest University of Technology and Economics, Budapest, Hungary \\ 3 Electrophysics Department, National Chiao-Tung University, HsinChu, Taiwan, R.O.C. \\ 4 Institut für Nanotechnologie, Forschungszentrum Karlsruhe, 76026 Karlsruhe, Germany
}

(Dated: October 28, 2018)

\begin{abstract}
We study numerically and analytically the dynamical (AC) conductance through a two-dot system, where only one of the dots is coupled to the leads but it is also side-coupled to the other dot through an antiferromagnetic exchange (RKKY) interaction. In this case the RKKY interaction gives rise to a "two-stage Kondo effect" where the two spins are screened by two consecutive Kondo effects. We formulate a renormalized scaling theory that captures remarkably well the cross-over from the strongly conductive correlated regime to the low temperature low conductance state. Our analytical formulas agree well with our numerical renormalization group results. The frequency-dependent current noise spectrum is also discussed.
\end{abstract}

PACS numbers: $\mathbf{7 5 . 2 0 . H r , 7 4 . 7 2 . - h}$

Introduction. The Kondo effect ${ }^{1}$ in semiconductor quantum dots has attracted significant theoretical and experimental interest in recent years. ${ }^{2,3,4}$ In a dot that is only weakly coupled to leads, charge fluctuations are typically suppressed due to Coulomb blockade ${ }^{5}$. However, if the dot has an odd number of electrons then the spin of this electron interacts antiferromagnetically with the spin of the conduction electrons in the leads, and at low temperatures it is screened through a Kondo effect. The formation of this Kondo state typically leads to an enhancement of the conductance at low bias voltages.

Recently, in the quest for designing multiple quantum dot systems with tunable spin control, which can be used in spintronics and quantum information processing, double quantum dots have become the focus of interest 6,7 In these systems, when both dots are tuned to the single spin regime, an effective spin-spin interaction known as the Ruderman-Kittel-Kasuya-Yoshida (RKKY) ${ }^{8}$ interaction is mediated between the two dots by the conduction electrons. $\underline{6}$ This RKKY coupling competes with the Kondo effect in these systems. In the case where the two dots are coupled to two separate electrodes, an antiferromagnetic RKKY interaction leads to a crossover between the Kondo and RKKY regimes $6,9,10,11,13$ : a sufficiently strong antiferromagnetic RKKY coupling will lock the spins of the two dots into a singlet, and thereby suppress the Kondo effect, while the Kondo effect persists for weak RKKY interactions. This picture is slightly modified under non-equilibrium conditions: Then, even for strong RKKY interaction, the Kondo effect is partially restored by the finite bias voltage allowing for triplet excitations. 12 Part of the rich physics has been studied previously in the framework of two impurity Kondo $\frac{14}{4}$ and Anderson impurity ${ }^{15}$ models, and the singlet-triplet cross-over has also been recently studied experimentally by Craig et al.,$\frac{6}{6}$ who observed a the
Kondo resonance for weak RKKY couplings and a splitting of the Kondo resonance for large RKKY interactions. Significant theoretical and experimental effort has been devoted to the Kondo-RKKY transition in these double quantum dot systems, and related multi-orbital systems with two Kondo-screening channels have also been studied extensively in recent years by different theoretical approaches. 16

Rather than studying the usual singlet-triplet transition itself, in the present paper we shall focus on an even simpler but equally interesting arrangement, where only one of the dots is coupled to external leads, but the two dots are still side-coupled to each other (see Fig. 1). Unlike the usual four lead set-up, where the cross-over between an RKKY and Kondo regimes is governed by a quantum critical point corresponding to a finite value of the RKKY coupling $J, 11,13$ in this side-coupled system the side-coupled spin is always screened for any positive $J$, and a Kosterlitz-Thouless-type quantum phase transition and a two-stage Kondo effect occurs, 17,18 We remark that this transition is essentially the two-dot analogue of

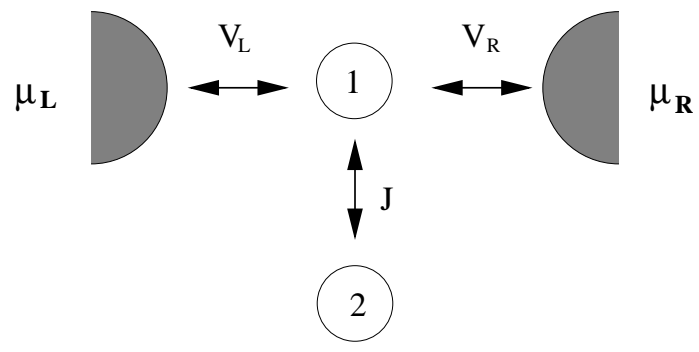

FIG. 1: Sketch of the two side-coupled quantum dots. Electrons tunnel from only one of them to the leads and they interact through an effective exchange interaction, $J$. 
the singlet-triplet quantum phase transition found in single dot devices, when the two dot levels are coupled to a single conduction electron mode in the leads 19,20

Although many interesting results have been obtained recently for this side-coupled system, $\stackrel{17,18}{\stackrel{1}{1}}$ there is still a lack of a detailed analytical and numerical understanding of the two-stage Kondo screening processes in the vicinity of the quantum phase transition. The main goal of the present work is to have some more detailed theoretical control and understanding of the transport properties of this transition. We shall reach this goal by combining numerical renormalization group 21 and renormalized perturbative scaling approaches. As we shall see, the latter relatively simple analytical framework is able to account for the numerical results over a wide range of energy scales, and together with Fermi liquid theory, it provides a reliable theoretical framework to understand the low energy cross-over.

We mostly focus on the T-matrix, but also present results on the linear $\mathrm{AC}$ conductance and the equilibrium current fluctuations. Both of these quantities can be observed experimentally and show features characteristic of the two-stage Kondo effect.

The Model. For the numerical calculations we shall describe the two side-coupled dots in Fig. 1 by an Andersonlike model. The two isolated dots are described by the Hamiltonian

$$
H_{D D}=\sum_{i=1,2} \frac{U_{i}}{2}\left(N_{i}-n_{g i}\right)^{2}+J \mathbf{S}_{1} \mathbf{S}_{2},
$$

where $i=1,2$ labels the two dots, $N_{i}=\sum_{\sigma} d_{i \sigma}^{\dagger} d_{i \sigma}$ is the number of electrons occupying $\operatorname{dot} i$ and $\mathbf{S}_{i}=$ $(1 / 2) \sum_{\sigma \sigma^{\prime}} d_{i \sigma}^{\dagger} \sigma_{\sigma \sigma^{\prime}} d_{i \sigma^{\prime}}$ is their spin. Each dot is subject to a charging energy, $U_{1} \approx U_{2}=U=E_{C}$, and the two dots are coupled by an exchange coupling, which is assumed to be antiferromagnetic, $J>0$. The $n_{g i}$ in Eq. (11) denote dimensionless gate voltages that set the occupation numbers, $\left\langle N_{i}\right\rangle$. In the rest of the paper, we restrict ourself to the case of particle-hole symmetry, $n_{g 1}=n_{g 2}=1$. However, this assumption is expected to be unimportant as long as $n_{g 1} \approx n_{g 2} \approx 1$.

The coupling of dot 1 to the leads is modeled by the usual tunneling Hamiltonian,

$$
H_{t}=\sum_{\alpha=L, R} \sum_{\epsilon, \sigma}\left(V_{\alpha} c_{\alpha \epsilon \sigma}^{\dagger} d_{1, \sigma}+\text { h.c. }\right) .
$$

Here, $V_{L}$ and $V_{R}$ denote the tunneling amplitudes to the left and right leads, respectively, and $c_{\alpha \in \sigma}^{\dagger}$ creates an electron in lead $\alpha=L, R$ with spin $\sigma$ and energy $\epsilon$. This tunnel coupling leads to a broadening of the level on $\operatorname{dot} 1$, the width of which is given by $\Gamma=\Gamma_{L}+\Gamma_{R}=$ $2 \pi\left(V_{L}^{2} \varrho_{L}+V_{R}^{2} \varrho_{R}\right)$, with $\varrho_{L / R}$ the density of states in the leads.

$A C$ conductance and noise: Our main goal is to determine $\mathrm{AC}$ transport properties in the linear response regime and see how the two-stage effect appears in these quantities. Fortunately, since there is no charge transfer between the two dots, the derivation of Ref. 22 carries over to our case, and the real part of the optical conductance is simply given by

$$
G^{\prime}(\omega)=\frac{G_{0}}{4 \omega} \sum_{\sigma} \int d \omega^{\prime}\left[f\left(\omega^{\prime}-\omega\right)-f\left(\omega^{\prime}+\omega\right)\right] T_{\sigma}\left(\omega^{\prime}\right),
$$

where $T(\omega)$ is "the transmission probability" at energy $\omega$, and

$$
G_{0}=\frac{2 e^{2}}{h} \frac{4 \Gamma_{L} \Gamma_{R}}{\left(\Gamma_{L}+\Gamma_{R}\right)^{2}}
$$

denotes the maximum conductance through the dot. The transmission coefficient $T_{\sigma}(\omega)$ appearing in Eq. (3) can be expressed as

$$
T_{\sigma}(\omega)=-\Gamma \operatorname{Im} G_{11 \sigma}(\omega),
$$

where we have introduced the retarded Green's function on dot 1: $G_{11 \sigma}(t)=-i \theta(t)\left\langle\left\{d_{1 \sigma}(t), d_{1 \sigma}^{\dagger}\right\}\right\rangle$. This Green function can be computed accurately using numerical renormalization group methods, $\stackrel{21}{\rightleftharpoons}$ but as we shall see, substantial analytical progress can be made by a special version of renormalized perturbation theory. Although Eq. (5D) is valid at any temperature, in the following we shall focus our attention to the high frequency regime, $\omega \gg T$, and set the temperature to zero, $T=0$.

The noise spectrum of the device is also of experimental relevance. This is defined as

$$
C(\omega)=\int_{-\infty}^{\infty} d t e^{i \omega t}\left[\langle I(0) I(t)\rangle-\langle I\rangle^{2}\right]
$$

At equilibrium, this is simply related to the linear conductance $G^{\prime}(\omega)$ by the fluctuation-dissipation theorem, 22

$$
C(\omega)=\frac{2 \hbar \omega}{\exp (\hbar \omega / k T)-1} G^{\prime}(\omega) .
$$

This formula simplifies further at $T=0$ temperature to $C(\omega)=2 \hbar|\omega| G^{\prime}(\omega) \theta(-\omega)$. Clearly, to determine the equilibrium noise spectrum of the device and its $\mathrm{AC}$ conductance only the Green's function of the first dot needs to be determined.

Renormalized perturbative scaling: Before presenting our numerical results, let us reach some analytical understanding of the physics of the side-coupled dot in the limit $J \rightarrow 0$. In this regime, "two-stage Kondo screening" takes place $\frac{17}{}$ : In the first stage, the spin of the dot 1 gets screened below the Kondo temperature $T_{K} \approx D e^{-\pi U / \Gamma}$, where the high energy cut-off $D$ denotes the effective half bandwidth of the conduction electrons. ${ }^{26}$ Clearly, for the Kondo effect to take place $J \ll T_{K}$ is required, otherwise the two spins are locked together to a singlet before the Kondo effect can take place. Then below the Kondo scale $T_{K}$ the electron on the first dot is dissolved in the conduction electron sea of the leads, and presents an effective fermionic bath for the electron on the second dot. Since the coupling between the two dots is antiferromagnetic, 


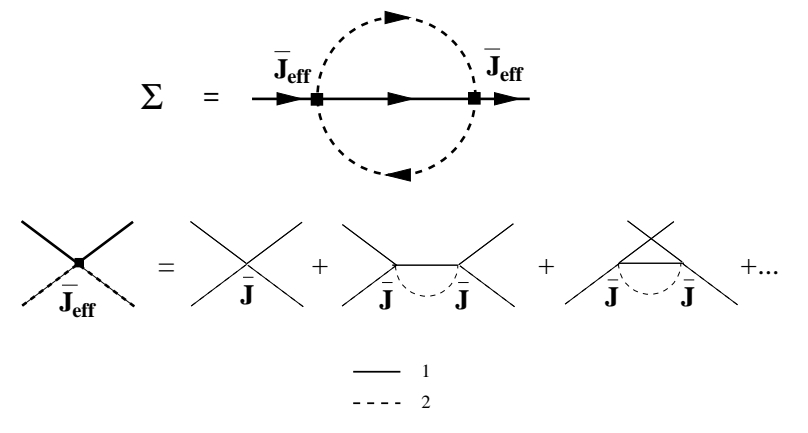

FIG. 2: Top: leading logarithmic contribution to the self energy of the first dot's Green's function. Solid lines represents the propagator $G_{d 1}^{0}$ of quantum dot 1, given by Eq. (8), while dashed lines denote the pseudofermion propagator associated with the side-coupled dot's spin, $S_{2}$. Full squares stand for the full leading logarithmic vertex function, given in the lower part of the figure. Crossings of dashed and continuous lines correspond to an interaction through the renormalized RKKY interaction, $J_{\text {eff }}=\tilde{J} / z$, given by Eq. (9).

another Kondo effect shall take place at a much smaller energy scale, $T^{\star}$, where the spin of the second dot is also screened. Our aim is to understand the formation of this second Kondo singlet in detail.

We first observe that in the regime of interest, $\omega \ll T_{K}$ the Matsubara Green's function of dot 1 can be approximated by the resonant level expression, $\underline{1}$

$$
G_{d 1}^{0}\left(i \omega_{n}\right)=\frac{z}{i \omega_{n}+i \tilde{T}_{K} \operatorname{sgn}\left(\omega_{n}\right)}
$$

where $z=c \frac{T_{K}}{\Gamma}$ denotes the quasiparticle weight at the Fermi energy, and $\tilde{T}_{K}=z \Gamma=c T_{K}$ is an energy of the order of the Kondo temperature, $T_{K}$. The precise value of the universal constant $c$ relating $T_{K}$ and $\tilde{T}_{K}$ depends on the definition of $T_{K}$. Throughout this paper we shall define $T_{K}$ as the half-width of the transmission $T(\omega)$. Then from fitting the NRG data we get $c \approx 0.5$. Note that the Lorentzian representing the Kondo resonance has a very small spectral weight, $z \ll 1$, and most of the spectral weight goes to the Hubbard peaks.

Fermi liquid theory and the basic principles of renormalization group also imply that the exchange interaction between the dot spins is renormalized by the same $z$-factor, i.e., in the regime $\omega \ll T_{K}$ the effective RKKY interaction reads

$$
H_{\mathrm{RKKY}} \rightarrow \frac{\tilde{J}}{z} \mathbf{S}_{1} \mathbf{S}_{2}
$$

In a first approximation one would think that, $\tilde{J}=J$, however, the fact that $\operatorname{Im} G_{d 1}$ has a large logarithmic tail above $T_{K}$ leads to a slight renormalization of this relation. From a fitting of the numerical data shown later, we obtain the approximate relation $\tilde{J} \approx 1.1 J$.

We are now in the position to develop a perturbative scaling theory in the small coupling, $\tilde{J}$. To do this, we used Abrikosov's pseudofermion representation to compute the second order self energy and vertex corrections, shown in in Fig 2. The dimensionless vertex function is given by the following expression:

$$
\gamma(\omega) \equiv \varrho(\omega) \Gamma(\omega)=\hat{\varrho}(\omega) \tilde{J}+(\hat{\varrho}(\omega) \tilde{J})^{2} \log \left(\frac{\tilde{T}_{K}}{-\omega}\right) \ldots
$$

where $\tilde{T}_{K}=c T_{K}$ is the effective width of the Kondo resonance, $\omega$ is the energy of the incoming electron, and $\hat{\varrho}(\omega)$ denotes the rescaled effective density of states of dot 1 , serving as a many-body reservoir for dot 2 ,

$$
\hat{\varrho}(\omega) \equiv \varrho(\omega) / z=\frac{\tilde{T}_{K}}{\pi\left(\omega^{2}+\tilde{T}_{K}^{2}\right)} .
$$

Eq. (10) is only of logarithmic accuracy, irrelevant terms of order $\omega / \tilde{T}_{K}$ have been neglected.

The Kondo temperature $T_{K} \sim \tilde{T}_{K}$ appears in Eq. (10) as a high energy cut-off. We can therefore perform a scaling transformation by reducing this cut-off, $\tilde{T}_{K} \rightarrow \tilde{T}_{K}^{\prime}$, and requiring the invariance of the vertex function for frequencies $\omega<\tilde{T}_{K}$ at the same time. This transformation sums up all leading logarithmic diagrams and leads to the following scaling equation

$$
\frac{d(\hat{\varrho}(\omega) \tilde{J})}{d l}=(\hat{\varrho}(\omega) \tilde{J})^{2}
$$

with the scaling variable defined as $l \equiv \log \left(\tilde{T}_{K} / \tilde{T}_{K}^{\prime}\right)$. Integrating this differential equation up to $l \equiv \log \left(\tilde{T}_{K} / \omega\right)$, one obtains the dimensionless vertex function in the leading logarithmic approximation:

$$
\gamma\left(\omega, \tilde{T}_{K}\right)=\frac{\hat{\varrho}(\omega) \tilde{J}}{1+\hat{\varrho}(\omega) \tilde{J} \log \left(-\omega / \tilde{T}_{K}\right)} .
$$

This equation can be rewritten with a little algebra as

$$
\gamma\left(\omega, \tilde{T}_{K}\right)=\frac{1}{\frac{\omega^{2}}{\tilde{T}_{K}^{2}} \log \frac{\tilde{T}_{K}}{T^{*}}+\log \frac{-\omega}{T^{*}}},
$$

with the second scale $T^{*}$ defined as

$$
T^{*}=\tilde{T}_{K} \exp \left(-\pi \tilde{T}_{K} / \tilde{J}\right)
$$

This scale also appears in the slave boson approach of Ref. 17, however, the latter approach does not account for the logarithmic corrections, which are of our main interest here. Clearly, the dimensionless vertex diverges at an energy, $\omega \approx T^{*}$, implying that the effective RKKY interaction becomes dominant below this scale and diverges in the limit $\omega \rightarrow 00^{21}$ In other words, below $T^{*}$ a singlet is formed from the two dot spins, and the scale $T^{*}$ can thus be viewed as the effective singlet-triplet binding energy. Note that the spin-singlet is always the ground state for any arbitrary RKKY coupling $J>0$.

The point $\tilde{J}=0$ is special: It separates the ferromagnetic phase $(J<0)$ from the antiferromagnetic phase 
discussed so far $(J>0)$. While in the antiferromagnetic phase the two dot spins are locked into a singlet, in the ferromagnetic phase the spins of the dots are bound to a triplet, which is then partially screened by the lead electrons, and correspondingly, the ground state is a doublet for $J<0$. Thus for $J<0$ the side-coupled system has a residual entropy. At the critical point, $J=0$, the scale $T^{*}$ diverges exponentially, corresponding to a KosterlitzThouless phase transition between these two states $\underline{\underline{17}, 18}$

The second order self-energy correction to the retarded Green's function $G_{d 1}^{0}$ simply gives the expression

$$
\Sigma(\omega)=S(S+1) \frac{\tilde{J}^{2}}{4 z} \frac{1}{\omega+i \tilde{T}_{K}} .
$$

where $\mathrm{S}=1 / 2$. Note that this correction scales as $\sim$ $J^{2} /\left(T_{K} z\right)$ and, although it looks to be very large at a first sight, it is actually small compared to $\left(G_{d 1}^{0}\right)^{-1} \sim$ $T_{K} / z$ as long as $J \ll T_{K}$. In the leading approximation, this self-energy results in the following Green's function:

$$
\Gamma G_{d}^{(2)}(\omega)=\frac{\tilde{T}_{K}}{\omega+i \tilde{T}_{K}-\frac{\bar{J}^{2} S(S+1)}{4} \frac{1}{\omega+i \tilde{T}_{K}}}
$$

Summing up the leading logarithmic corrections to the self-energy simply amounts to replacing $\tilde{J}$ in Eq. (17) by $\gamma(\omega) / \hat{\varrho}(\omega)$, and thereby results in the following transmission coefficient,

$$
T_{\sigma}(\omega)=-\operatorname{Im}\left\{\frac{\tilde{T}_{K}^{3}}{\omega\left(\tilde{T}_{K}^{2}-\frac{1}{4} S(S+1) \pi^{2}\left(\omega^{2}+\tilde{T}_{K}^{2}\right) \gamma^{2}(\omega)\right)+i \tilde{T}_{K}\left(\tilde{T}_{K}^{2}+\frac{1}{4} S(S+1) \pi^{2}\left(\omega^{2}+\tilde{T}_{K}^{2}\right) \gamma^{2}(\omega)\right)}\right\} .
$$

The logarithmic corrections hidden in $\gamma$ result in the formation of a dip in the spectral density of dot 1, corresponding to a suppression of the transmission coefficients at energies $\omega \sim T^{*} \ll T_{K}$. This dip in $T_{\sigma}(\omega)$ also implies the appearance of a dip in the $\mathrm{AC}$ conductance discussed later, and is a clear signature of the formation of a singlet ground state. Physically, it is a consequence of the fact that electrons promoted from one side of the device to the other must first break up the singlet of energy $T^{*}$ formed by the two dot spins. Clearly, electrons of energy $\omega<T^{*}$ are not energetic enough to break up this singlet and therefore their transport is suppressed.

The logarithmic approximation breaks down below $\omega \sim T^{*}$. There a Fermi liquid is formed and $T_{\sigma}(\omega)$ scales as

$$
T_{\sigma}(\omega) \approx a+b \frac{\omega^{2}}{\left(T^{*}\right)^{2}}, \quad\left(\omega \ll T^{*}\right) .
$$

The constant $a$ vanishes for electron-hole symmetry and remains typically small unless electron-hole symmetry is dramatically broken, while the coefficient $b$ is a number of the order of unity.

Comparison with NRG. The transport properties of the side-coupled quantum dot system can also be studied numerically by Numerical Renormalization Group (NRG) methods. The transmission coefficient $T(\omega)$ through dot 1 obtained from NRG at different RKKY couplings $J$ is plotted in Fig 3, In all figures we compensated for a $6 \%$ loss of the spectral weight. The large resonance is a manifestation of the Kondo effect displayed by the first dot, and the sharp dips in the transmission at $\omega \approx 0$ are due to the formation of the singlet state below the energy $T^{*}$.

The numerically obtained transmission coefficients are compared to the analytical formula (18) in Fig. 4. The perturbative expression agrees well with the numerical results over a wide range of energy scales between $T^{*}$ and $T_{K}$. It is interesting to observe the deviations above $T_{K}$, where the simple Lorentzian approximation we made for $G_{d}^{(0)}$ fails to account for the fact that the resonance on dot 1 is also a Kondo resonance. This Lorentzian approximation thus completely neglects the large logarithmic tails for $\omega>T_{K}$. In the inset we also show the scale $T^{*}$ as extracted from our fits as a function of $1 / J$. The extracted scales compare very well with the analyt-

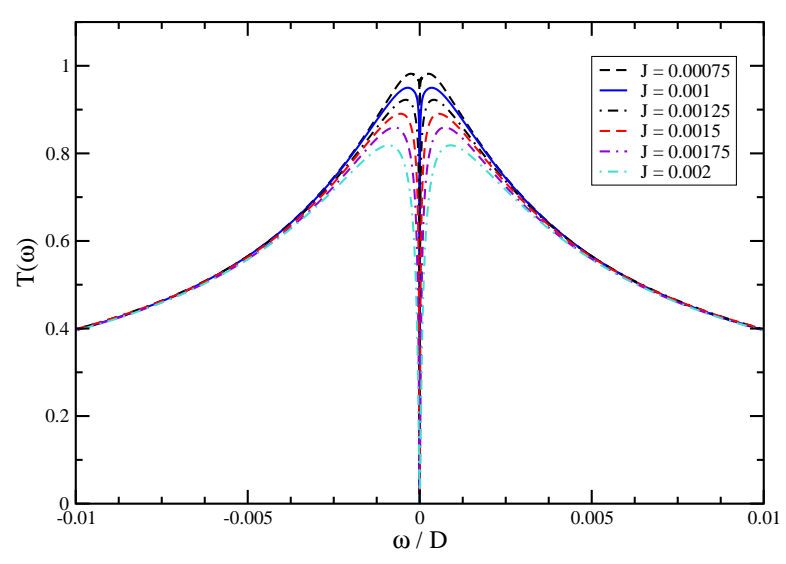

FIG. 3: Transmission coefficient through dot 1 at zero temperature for different RKKY couplings, $J$. The energy unit is the half bandwidth, $D=1$. The Anderson model's parameters were $U=1, \epsilon_{d 1}=\epsilon_{d 2}=-0.5, \Gamma_{1 L}=\Gamma_{1 R}=0.1$, resulting in a Kondo temperature of $T_{K} \approx 0.0055$. We used a discretization parameter $\Lambda=2$. 
ical expression, Eq. (15), indicated by the solid line.

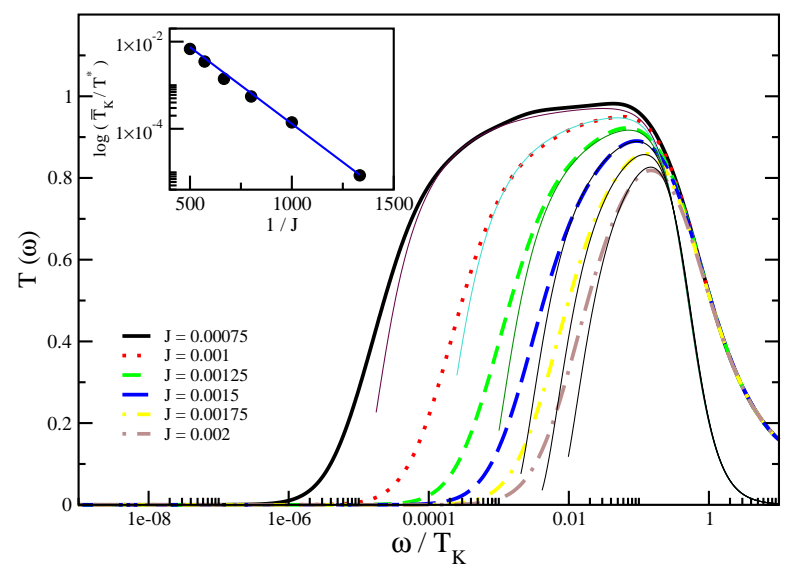

FIG. 4: Fit of the numerically obtained transmission coefficient $T(\omega)$ by the perturbative expression, (18) for various values of $\tilde{J}$. For all fits we used $\tilde{T}_{K}=0.5 T_{K}=0.003$. The inset shows the scale $T^{*}$ extracted from the fit, as a function of $1 / J$.

The two-stage Kondo effect and the K-T transition can also be observed in the $\mathrm{AC}$ conductance of the two-dot device or the noise spectrum. Such measurements have been indeed done in recent experiments of high-frequency current fluctuations ${ }^{23}$, though the linear $\mathrm{AC}$ conductance measurements in the relevant frequency regime are very difficult due to background currents from the

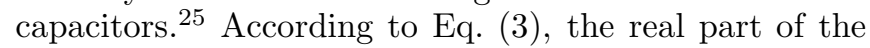
conductance through the device can be computed from the transmission coefficient through a simple integration. The resulting curves are displayed in Fig. 5 .

It is interesting to remark that the low energy crossover of the transmission coefficient and the conductance is described by universal cross-over functions for $\omega, T^{*} \ll$ $T_{K}$. The conductance, e.g., is approximately given by

$$
G^{\prime}(\omega) \approx G_{0} g\left(\omega / T^{*}\right)
$$

in this regime. Here the scaling function $g$ depends somewhat on electron-hole symmetry breaking, but in case of electron-hole symmetry it is completely universal. Then for very small frequencies it scales to zero as $g(\omega) \approx 0.06\left(\omega / T^{*}\right)^{2}$, while at high energies it approaches 1 logarithmically, $g\left(\omega / T^{*}\right) \approx 1-\alpha / \log ^{2}\left(\omega / T^{*}\right)$. This universal cross-over function can be extracted from the NRG results, and is displayed in Fig. 6. The transmission coefficient $T_{\sigma}(\omega)$ displays similar universal scaling properties.

Finally, let us discuss the current noise at zero temperature, plotted in Fig 7, At $T=0$ temperature $C(\omega)$ has only weight for $\omega<0$. For $T^{*}<-\omega<T_{K}$ we have $C(\omega) \propto|\omega|$ corresponding to the Fermi-liquid property of a perfectly transmitting quantum dot ${ }^{22}$ However, for $-\omega<T^{*}$ this behavior crosses over to a power law scaling, where in case of electron-hole symmetry one has

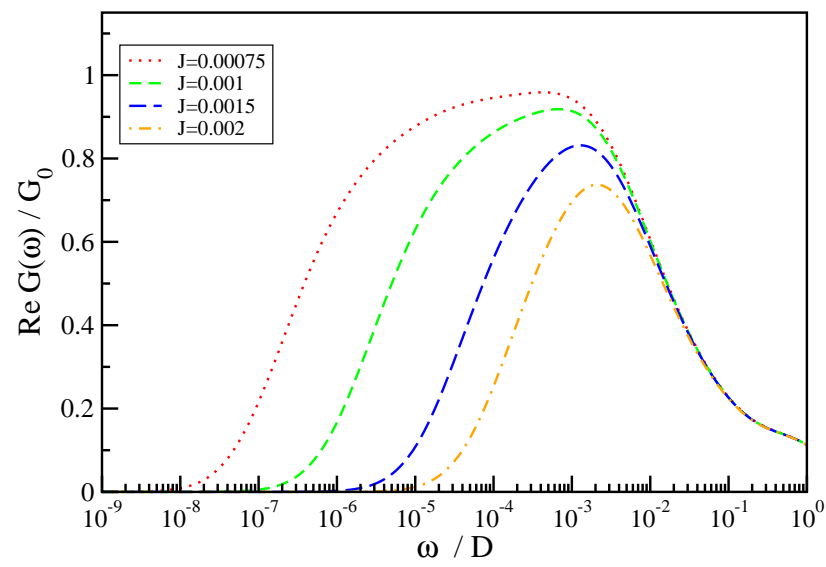

FIG. 5: AC conductance normalized by $G_{0}$ for the same parameters as in Fig. 3 .

$C(\omega) \propto|\omega|^{3} /\left(T^{*}\right)^{2}$. This behavior is somewhat modified once electron-hole symmetry is broken. Then the conductance remains finite even in the $\omega \rightarrow 0$ limit, and correspondingly, another cross-over may take place from the $C(\omega) \propto|\omega|^{3}$ regime to a linear regime, $C(\omega) \propto|\omega|$ at some energy $T^{* *} \ll T^{*}$.

Conclusions. We provided a complete analytical and numerical analysis of the $\mathrm{AC}$ transport properties and the two-stage Kondo screening in side-coupled quantum dots. Our analytical results were based on renormalized perturbation and scaling theory, and they agree well with the NRG result over a wide frequency range. We also determined the linear AC conductance and the equilibrium current noise which can both be measured experimentally and reflect the two-stage Kondo effect and the K-T

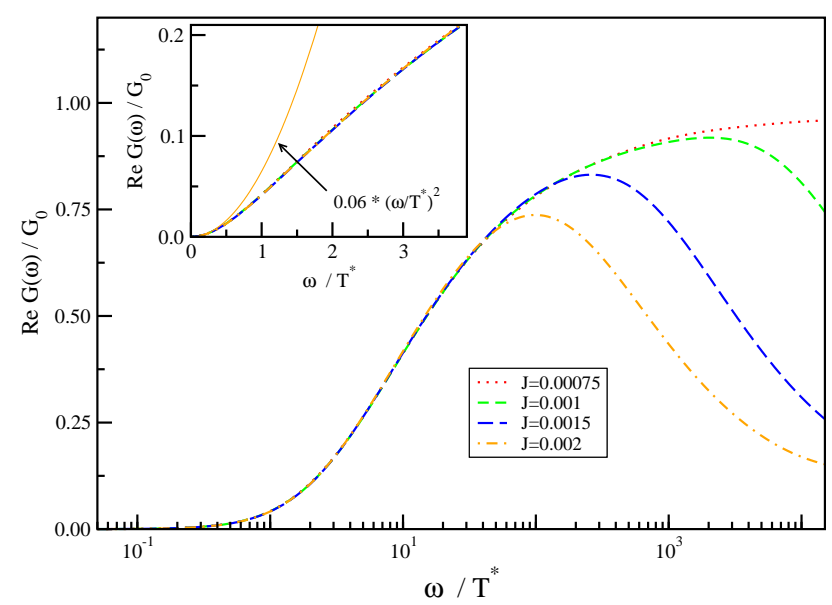

FIG. 6: $G^{\prime}(\omega)$ as a function of $\omega / T^{*}$ for various values of $J$. Below the Kondo scale all curves collapse to a universal curve. Inset: small frequency part of this universal curve on a linear scale. We also show the quadratic behavior characteristic of electron-hole symmetry, determined from a log-log fit of the curves. 


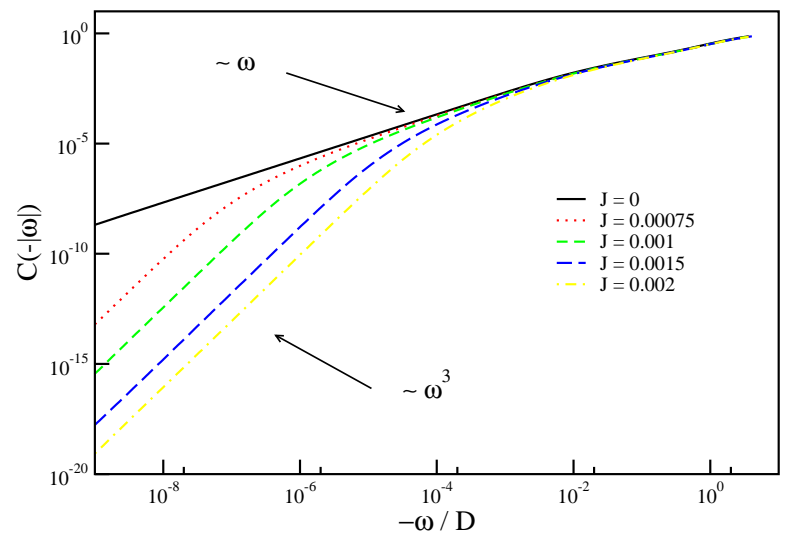

FIG. 7: Equilibrium current noise $C(\omega)$ in case of electronhole symmetry for $\omega<0 . C(\omega)$ exhibits linear dependence on $\omega$ for $T^{*}<|\omega|<T_{K}$, that crosses over to a cubic behavior below $T^{*}$. Note that $C(\omega)=0$ for $\omega>0$. The parameters are the same as in Fig 3 transition. We also computed the universal cross-over functions that describe the emergence of the triplet state at the energy $T^{*}$.

Acknowledgments. This work has been supported by the DFG-Center for Functional Nanostructures and by the Virtual Institute for Research on Quantum Phase Transitions at the University of Karlsruhe. C.H.C acknowledges the support from the NSC and the MOE ATU Program of Taiwan, R.O.C. G.Z. has been supported by the Humboldt Foundation and by Hungarian grants, OTKA Nrs. NF061726, and T046303. He also acknowledges the hospitality of the C.A.S., Norway, where part of this research has been done.
1 A.C. Hewson, The Kondo problem to Heavy Fermions (Cambridge University Press, Cambridge, UK, 1997).

2 L. Kouwenhoven and L. Glazman, Physics World 14, 33 (2001).

3 D. Goldhaber-Gordon et al., Nature 391, 156 (1998); S.M. Cronenwett et al., Science 281, 540 (1998); F. Simmel et al., Phys. Rev. Lett. 83, 804 (1999); J. Schmid et al., Phys. Rev. Lett. 84, 5824 (2000); W. G. van der Wiel et al., Science 289, 2105 (2000).

4 L.I. Glazman and M.E. Raikh, Sov. Phys. JETP Lett. 47, 452 (1988); T.K. Ng, P.A. Lee, Phys. Rev. Lett. 61, 1768 (1988).

5 L.P. Kouwenhoven et al. in Mesoscopic Electron Transport, eds. L.L. Sohn, L.P. Kouwenhoven, and G. Schön, NATO ASI Series E, vol. 345, pp. 105-214 (Kluwer, Dordrecht, 1997).

${ }^{6}$ N. J. Craig, J.M. Taylor, E.A. Lester, C.M. Marcus, M.P. Hanson,

and A.C. Gossard, Science 304, 565 (2004).

7 H. B. Heersche, Z. de Groot, J.A. Folk, L.P. Kouwenhoven, H.S.J. van der Zant, A.A. Houck, J. Labaziewicz, I.L. Chuang, Phys. Rev. Lett. 96, 017205 (2006).

8 M. A. Ruderman, C. Kittel, Phys. Rev. 96, 99 (1954); T. Kasuya, Prog. Theor. Phys. 16, 45 (1956); K. Yosida, Phys. Rev. 106, 893 (1957).

9 P. Simon, R. Loṕez, and Y. Oreg, Phys. Rev. Lett. 94, 086602 (2005).

10 M. G. Vavilov and L. I. Glazman, Phys. Rev. Lett. 94, 086805 (2005).

11 G. Zarand, C.H. Chung, P. Simon, M. Vojta, Phys. Rev. Lett. 97, 166802 (2006).

12 V. Koerting, P. Wölfle, and J. Paaske, Phys. Rev. Lett. , accepted for publication

13 C.H. Chung and W. Hofstetter, cond-mat/0607772.
14 C. Jayaprakash, H. R. Krishna-murthy and J. W. Wilkins, Phys. Lett. 47, 737 (1981); B. A. Jones, C. M. Varma, Phys. Rev. Lett. 58, 843 (1987); B. A. Jones, C. M. Varma, and J. W. Wilkins $i b d b .61,125$ (1988); B. A. Jones and C. M. Varma, Phys. Rev. B 40, 324 (1989); I. Affleck and A. W. W. Ludwig and B. A. Jones, Phys. Rev. B 52, 9528 (1995).

15 O. Sakai and Y. Shimizu, J. Phys. Soc. Japan, Vol. 61, No. 7, 2333 (1992); O. Sakai and Y. Shimizu, ibdb. Vol. 61 No. 7, 2348 (1992).

16 J. Kroha, S. Kirchner, G. Sellier, P. Wïfle, D. Ehm, F. Reinert, S. Huefner, C. Geibel, Physica E 18, 69 (2003).

17 P.S. Cornaglia and D.R. Grempel, Phys. Rev. B 71, 075305 (2005).

18 M. Vojta, R. Bulla, and W. Hofstetter, Phys. Rev. B 65, 140405 (2002).

19 W. Hofstetter and H. Schöller, Phys. Rev. Lett. 88, 061803 (2002);

${ }^{20}$ Kogan et al., Phys. Rev. Lett. 67, 113309 (2003).

21 K. G. Wilson, Rev. Mod. Phys. 47, 773 (1975); T.A. Costi, A.C. Hewson, and V. Zlatić, J. Phys.: Cond. Mat. 6, 2519 (1994); W. Hofstetter, Phys. Rev. Lett. 85, 1508 (2000).

22 M. Sindel, W. Hofstetter, J. von Delft, and M. Kindermann, Phys. Rev. Lett. 94, 196602 (2005).

23 R. Deblock, E. Onac, L. Gurevich, and L. P. Kouwenhoven, Science 301, 203 (2003).

24 A.-P. Jauho, N.S. Wingreen and Y. Meir, Phys. Rev. B 50, 5528 (1994).

25 P. Nordlander, M. Pustilink, Y. Meir, N.S. Wingreen, and D. C. Langreth, Phys. Rev. Lett. 83, 808 (1999).

26 This cut-off is typically provided by the level spacing $\delta \epsilon$ of the quantum dots.

27 Y. Meir, N.S. Wingreen, and P.A. Lee, Phys. Rev. Lett. 70, 2601 (1993). 